\title{
Creep properties of a new Re free single crystal Ni-based superalloy, NKH71
}

\author{
Yoshihiro Kondo $^{1, \text { a }}$, Yuusuke Kubo ${ }^{1}$, Nobuhiro Miura ${ }^{1}$, Yoshinori Murata ${ }^{2}$, and Akira Yoshinari ${ }^{3}$ \\ ${ }^{1}$ National Defense Academy, Department of Mechanical Systems Engineering, 1-10-20 Hashirimizu, Yokosuka, Japan \\ 2 Nagoya University, Department of Materials Science and Engineering, Graduate School of Engineering, Furo-cho, \\ Chikusa, Nagoya, Japan \\ ${ }^{3}$ Mitsubishi Hitachi Power Systems Ltd., 3-1-1 Sachi-machi, Hitachi, Japan
}

\begin{abstract}
The creep of the newly developed Ni-based, Re free, single crystal superalloy NKH71 was examined at $1273 \mathrm{~K}$. The creep properties were compared to those of CMSX-4, a second-generation commercial superalloy containing 3\%Re. When the (applied) stress was higher than $160 \mathrm{MPa}$, the rupture lives of the present alloy were shorter than those of CMSX-4. However, when the stress was below $160 \mathrm{MPa}$, the rupture lives of the present alloy became longer than those of CMSX-4. It was noted that this lower stress level was more important when Nibased superalloys were used for engineering land-based gas turbines. TEM and SEM observations showed that NKH71 formed a rafted structure in the early stage of creep. This was because a large number of dislocations existed in the $\gamma / \gamma^{\prime}$ interfaces before creep. In contrast, CMSX-4 had very few dislocations before creep. This observation explained why NKH71 was inferior to CMSX-4 under high stress. However under lower stress, the $\gamma / \gamma^{\prime}$ rafted structure changed to a more complex morphology in CMSX-4, while that of NKH71 tended to remain unchanged. This conservation of the rafted structure lead to the good creep resistance of NKH71 under a lower stress. In brief, the new alloy was shown to have good creep resistance even without Re element.
\end{abstract}

\section{Introduction}

Recently, turbine inlet gas temperature of land-based gas turbines have increased to improve thermal efficiency and to reduce $\mathrm{SO}_{\mathrm{x}}, \mathrm{NO}_{\mathrm{x}}$ and $\mathrm{CO}_{2}$ emissions [1]. Ni-based superalloys can be used for this purpose. The service conditions for this application are less severe than for aero applications: e.g. lower stress. Re-containing $\mathrm{Ni}$ superalloys, have been used for aero turbine blades, since these alloys showed improved creep resistance. However, undesirable topologically close packed (TCP) phases are formed at higher temperatures in this kind of alloy [2-4]. Moreover, Re is an expensive element. Thus, we aimed to eliminate Re from Ni-based superalloys. The present alloy, NKH71, was produced for this purpose [5]. Indeed, this alloy shows good creep resistance, equal to or better than conventional second-generation Re-containing alloys such as CMSX-4. The present paper reports creep properties of NKH71 at $1273 \mathrm{~K}$ in comparison with CMSX-4.

\section{Experimental procedure}

Single crystals of a newly developed Ni-based superalloy, NKH71, were cast as cylindrical bars of $13 \mathrm{~mm}$ in diameter and having an orientation within 5 degrees from [001]. Chemical compositions of the alloy are given in Table 1 with those of CMSX-4 which contained Re and was

\footnotetext{
${ }^{a}$ Corresponding author: kondo@nda.ac.jp
}

Table 1. Chemical compositions of NKH71 and CMSX-4 (wt/\%).

\begin{tabular}{|c|c|c|c|c|c|c|c|c|c|c|}
\hline & $\mathrm{Ti}$ & $\mathrm{Cr}$ & $\mathrm{Co}$ & $\mathrm{Mo}$ & $\mathrm{Ta}$ & $\mathrm{W}$ & $\mathrm{Re}$ & $\mathrm{Al}$ & $\mathrm{Hf}$ & $\mathrm{Ni}$ \\
\hline NKH71 & 1.2 & 12.2 & - & 0.5 & 5.8 & 7.8 & - & 5.0 & - & Bal. \\
\hline CMSX-4 & 0.9 & 6.4 & 9.3 & 0.6 & 6.2 & 6.2 & 2.8 & 5.5 & 0.1 & Bal. \\
\hline
\end{tabular}

used for comparison. The bars were solution treated at $1573 \mathrm{~K}$ for $2.88 \times 10^{4} \mathrm{~s}$ and double-aged at $1373 \mathrm{~K}$ for $1.44 \times 10^{4} \mathrm{~s}$ and at $1144 \mathrm{~K}$ for $7.2 \times 10^{4} \mathrm{~s}$. Specimens for tensile creep having a gauge diameter of $6 \mathrm{~mm}$ and length of $30 \mathrm{~mm}$ were machined from the bars. Creep tests were conducted at $1273 \mathrm{~K}$ under the stress of 100-400 MPa. The creep strain was recorded using linear variable differential transformers attached to the gauge section. Some creep tests were interrupted in order to see structural changes, using $8 \mathrm{~mm}$ diameter and $50 \mathrm{~mm}$ length specimens. A field emission scanning microscope (FE-SEM) and a transmission electron microscope (TEM) were used to see these changes. The observed planes were (100) and (001). Samples for SEM observation were metallographically polished and electro-etched using a saturated oxalic acid solution. Discs of $3 \mathrm{~mm}$ diameter were spark-cut from creep interrupted specimens and twin-jet polished for TEM observation using a mixture of perchloric and ethanol. The dislocation density was measured on $200 \mu \mathrm{m}^{2}$ from TEM images.

This is an Open Access article distributed under the terms of the Creative Commons Attribution License 4.0, which permits unrestricted use, distribution, and reproduction in any medium, provided the original work is properly cited. 


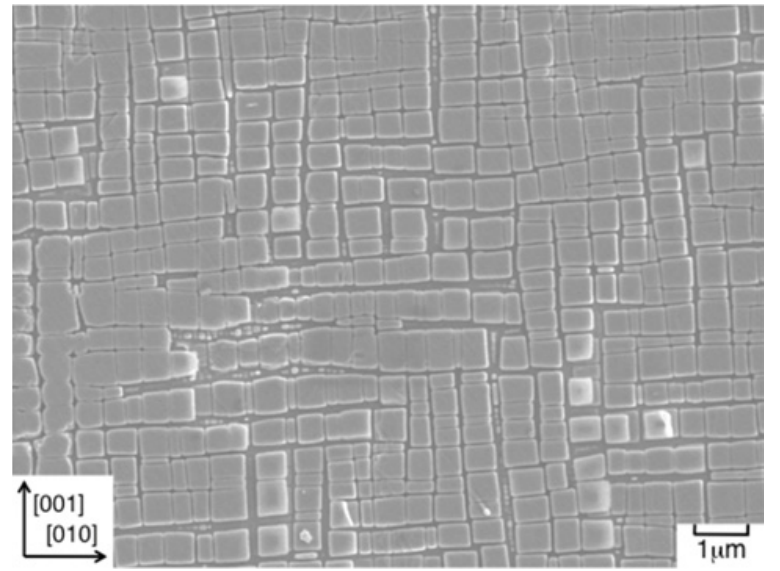

Figure 1. Scanning electron micrograph of the as-heat treated NKH71.

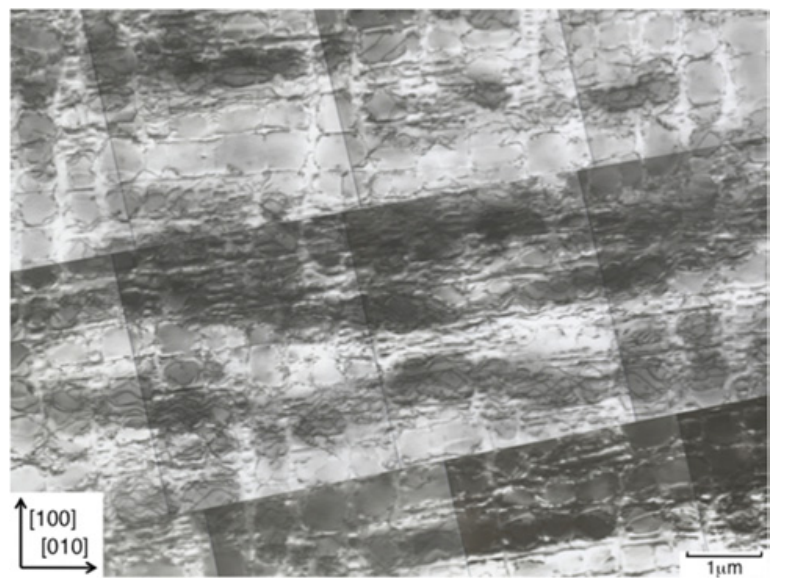

Figure 2. Transmission electron micrograph of the as-heat treated NKH71.

\section{Experimental result}

\subsection{The microstructure of as-heat treated NKH71}

A scanning electron micrograph is shown in Fig. 1. Cuboidal $\gamma^{\prime}$ precipitates are regularly arrayed. The average edge length of the $\gamma^{\prime}$ precipitates is $0.5 \mu \mathrm{m}$ and the width of average $\gamma$ channels is $0.1 \mu \mathrm{m}$. The volume fraction of $\gamma^{\prime}$ is estimated as $78 \%$. No eutectic $\gamma^{\prime}$ exists.

A transmission electron micrograph (TEM) is shown in Fig. 2. A large number of dislocations are observed in the $\gamma / \gamma^{\prime}$ interfaces even before creep deformation. Of course, no stacking faults exist in the cuboidal $\gamma^{\prime}$ precipitates.

\subsection{Stress-time to rupture curves}

The stress-time rupture curves at $1273 \mathrm{~K}$ are shown in Fig. 3, which also includes that of CMSX-4 for comparison. When the stress is over $200 \mathrm{MPa}$, the creep rupture lives of NKH71 are shorter than those of CMSX-4. However, when the stress is less than $160 \mathrm{MPa}$, NKH71 is stronger in creep rupture lives.

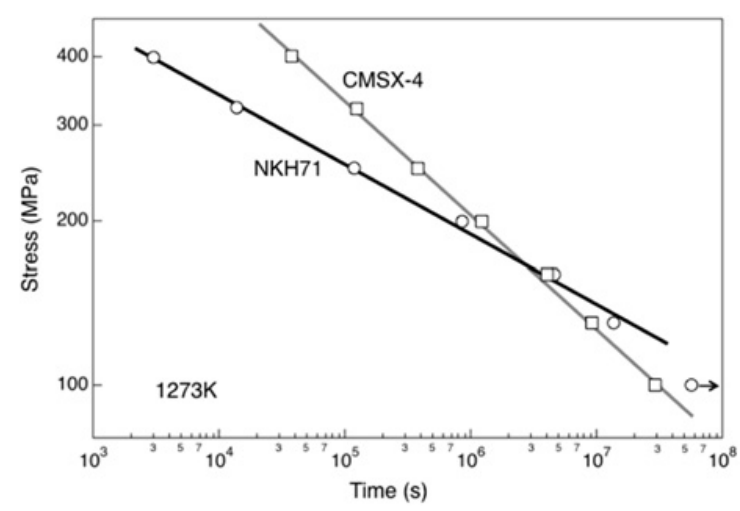

Figure 3. Stress-time to rupture curves of NKH71 and CMSX-4 at $1273 \mathrm{~K}$.
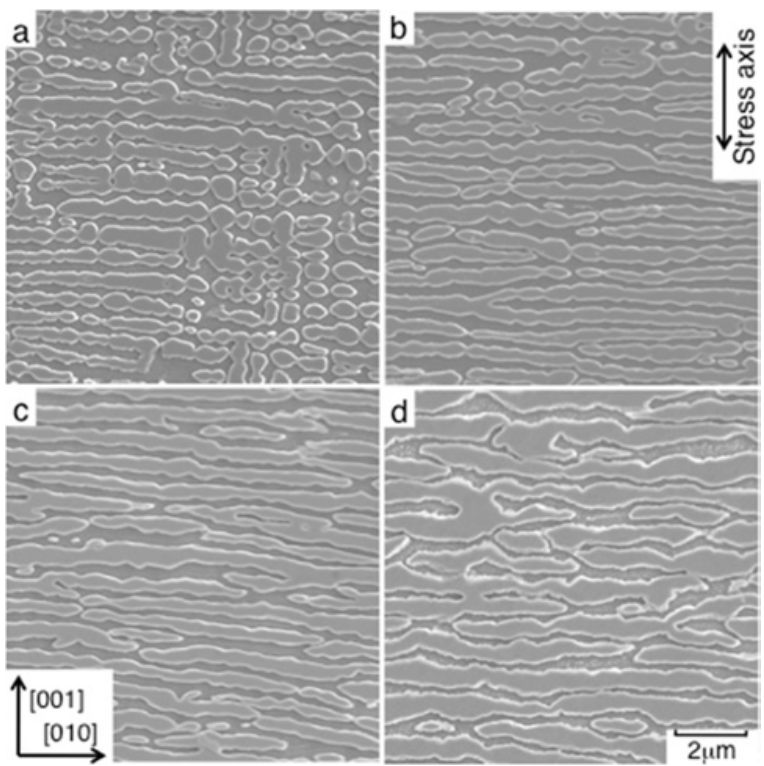

Figure 4. Scanning electron micrograph of the creep interrupted (a) $3.60 \times 10^{4}$, (b) $3.60 \times 10^{5}$, (c) $3.24 \times 10^{6} \mathrm{~s}$ ) and (d) ruptured specimens of NKH71 at $1273 \mathrm{~K}$ and $160 \mathrm{MPa}$.

\subsection{Morphological change of $\gamma^{\prime}$ precipitates with creep}

Scanning electron micrographs of NKH71 crept under the stress of $160 \mathrm{MPa}$ are shown in Fig. 4. When crept for $3.60 \times 10^{4} \mathrm{~s}$, some cuboidal $\gamma^{\prime}$ precipitates begin to connect with each other in the direction perpendicular to the stress axis (Fig. 4a). The rafted $\gamma / \gamma^{\prime}$ structures perpendicular to the stress axis are observed when crept for $3.60 \times 10^{5} \mathrm{~s}$ (Fig. 4b). When crept for $3.24 \times 10^{6} \mathrm{~s}$, the fully rafted $\gamma / \gamma^{\prime}$ structure is observed and the linear $\gamma / \gamma^{\prime}$ interfaces are clearly seen (Fig. 4c). This corresponds to the occurrence of the minimum creep rate. When creep ruptured, the $\gamma^{\prime}$ precipitates have begun to merge even in the vertical direction parallel to the stress axis (Fig. 4d).

The morphological change is presented quantitatively in Fig. 5, where the aspect ratio, the ratio of the length over the width of $\gamma^{\prime}$ precipitates, is plotted against the creep time for NKH71 and CMSX-4 [6]. Here, the results for 160 and $250 \mathrm{MPa}$ are shown. Before creep, the aspect 


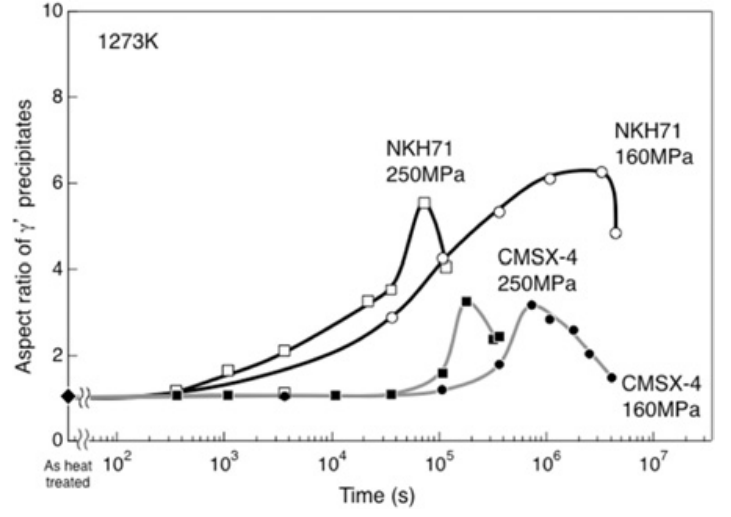

Figure 5. Changes in the aspect ratio of the $\gamma^{\prime}$ precipitates of NKH71 and CMSX-4 crept at 160 and 250 MPa with creep time.

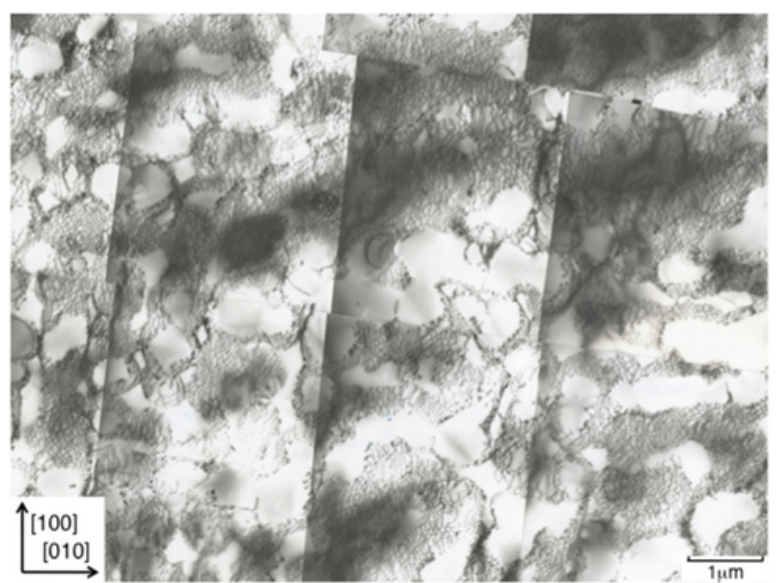

Figure 6. Transmission electron micrograph of interrupted creep specimen for $1.08 \times 10^{5} \mathrm{~s}$ at $1273 \mathrm{~K}$ and $160 \mathrm{MPa}$.

ratio is one. As the creep progressed, the aspect ratio increases with time, attains the maximum value and then decreases. The maximum aspect ratio is attained at a shorter time for $250 \mathrm{MPa}$ creep than for $160 \mathrm{MPa}$ creep. However, the maximum aspect ratio of $160 \mathrm{MPa}$ creep is larger. On the other hand, the aspect ratio of CMSX-4 does not change until the creep time of $1.0 \times 10^{5} \mathrm{~s}$. Then, the ratio increases and again decreases after attaining a maximum. The ratio of NKH71 is higher than that of CMSX-4 for all the creep times. In brief, the $\gamma / \gamma^{\prime}$ rafted structure is formed earlier in NKH71 than in CMSX-4. Also NKH71 keeps the $\gamma / \gamma^{\prime}$ rafted structure until rupture.

\subsection{Dislocation structure at $\gamma / \gamma^{\prime}$ interfaces}

Using TEM foils, parallel to (103), a transmission electron micrograph of the interrupted creep specimen of NKH71 for $1.08 \times 10^{5} \mathrm{~s}$ at $1273 \mathrm{~K}$ and $160 \mathrm{MPa}$ is shown in Fig. 6, where the incident beam direction, $\mathrm{B}$, is closed to [001]. A large number of dislocations are entangled with each other, existed at the $\gamma / \gamma^{\prime}$ interfaces in NKH71 even in the as-heat treated state. Few dislocations are observed in CMSX-4 before creep. No stacking faults are observed.

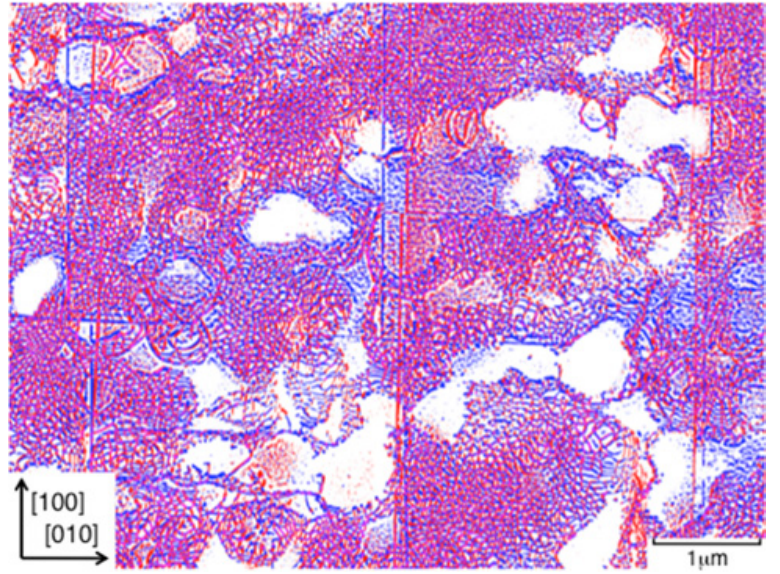

Figure 7. Superimposed image of transmission electron micrograph where $\mathrm{B}=[001], \mathrm{g}=[020]$ and [200], on the same region at the $\gamma / \gamma^{\prime}$ interfaces, by using the interrupted creep specimen for $1.08 \times 10^{5} \mathrm{~s}$ at $1273 \mathrm{~K}, 1600 \mathrm{MPa}$. $(\mathrm{g}=[020]:$ red, [200] : blue, [020]+[200] : purple).

\subsection{Quantitative examination of dislocations}

The dislocation density at the $\gamma / \gamma^{\prime}$ interfaces is regarded as the quantitative parameter showing the change in the dislocation substructures at the $\gamma / \gamma^{\prime}$ interfaces with creep time. In order to make the quantitative measurement of the dislocation density, it must be understood that there are two invisibility conditions on the basis of the $\mathrm{g} \cdot \mathrm{b}=0$ invisibility criterion. For example, when the TEM observations are performed with the incident beam directions, $\mathrm{B}=[001]$, and the g-vector, $\mathrm{g}=[020]$, dislocations with Burgers vectors of [101] and [10-1] are invisible, but are visible with $\mathrm{B}=[001], \mathrm{g}=[200]$. Hence the dislocation density cannot be accurately estimated by using only one g-vector. The TEM observations should be carried out with two different g-vectors on the same region and the images from each g-vector should be superimposed on the other to express the accurate dislocation substructures at the $\gamma / \gamma^{\prime}$ interfaces [7].

Using the invisibility condition of $\mathrm{g} \cdot \mathrm{b}=0$ and two types of g-vector, the dislocations in the $\gamma / \gamma^{\prime}$ interfaces are characterized and their density measured. An example of a TEM image used for this analysis is shown in Fig. 7 for a specimen crept for $1.08 \times 10^{5} \mathrm{~s}$ under the stress of $160 \mathrm{MPa}$. The accurate expression of all dislocations at the $\gamma / \gamma^{\prime}$ interfaces can be attained by the TEM observations with the two different $g$-vectors on the same area.

The change in the dislocation density, thus determined, at the $\gamma / \gamma^{\prime}$ interfaces with creep time at $160 \mathrm{MPa}$ is shown, in Fig. 8. Naturally, the density increases with creep time. Before creep, the density is $1.0 \times 10^{13} \mathrm{~m}^{-2}$ and it increases to $3.0 \times 10^{13} \mathrm{~m}^{-2}$ for the creep time of $3.6 \times 10^{4} \mathrm{~s}$. This density stays the same until rupture.

The change in the dislocation density at the $\gamma / \gamma^{\prime}$ interfaces with creep time at $250 \mathrm{MPa}$ is shown in Fig. 9. The dislocation density at the $\gamma / \gamma^{\prime}$ interfaces increases with increasing creep time until rupture. From these results, the change in the dislocation density at the $\gamma / \gamma^{\prime}$ interfaces with creep time at lower stress is different from that at higher stress. 


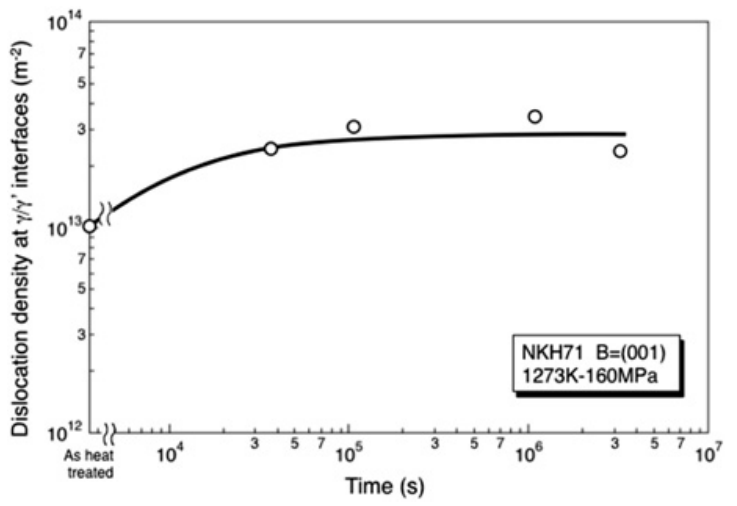

Figure 8. The change in the dislocation density at the $\gamma / \gamma^{\prime}$ interfaces with creep time at $1273 \mathrm{~K}$ and $160 \mathrm{MPa}$.

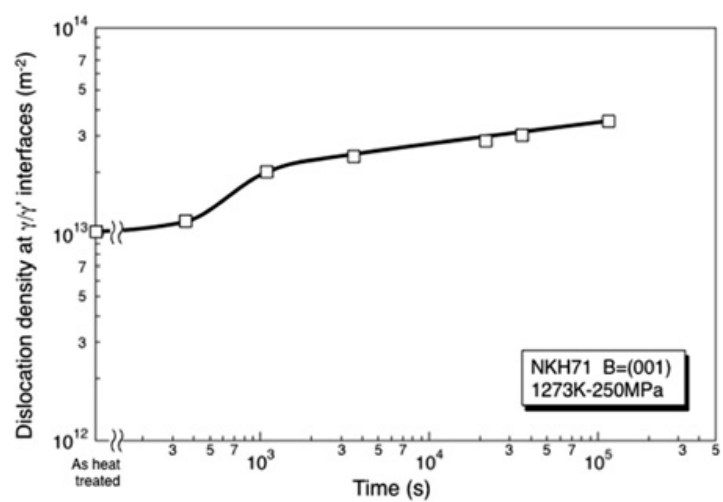

Figure 9. The change in the dislocation density at the $\gamma / \gamma^{\prime}$ interfaces with creep time at $1273 \mathrm{~K}$ and $250 \mathrm{MPa}$.

\section{Discussion}

As shown above, $\gamma^{\prime}$ precipitates show a rafted morphology earlier in NKH71 than in CMSX-4. This rafted $\gamma / \gamma^{\prime}$ structure is kept until rupture. The creep life of NKH71 is shorter than CMSX-4, when the stress is higher than $200 \mathrm{MPa}$. However, when the stress is below $160 \mathrm{MPa}$, the creep life of NKH71 is longer than that of CMSX-4. This result has been understood in terms of the dislocation structure in the $\gamma / \gamma^{\prime}$ interfaces, as described below.

The change in aspect ratios of the $\gamma^{\prime}$ precipitates of NKH71 and CMSX-4 with creep time under the stresses of 160 and $250 \mathrm{MPa}$ are shown in Fig. 10. The ratio of CMSX-4 at 160 and $250 \mathrm{MPa}$ increases with time and after reaching 3 , the ratio decreases. The ratio of NKH71 at $160 \mathrm{MPa}$ increases with time in the beginning and stays constant until rupture after attaining maximum of 6 until the $80 \%$ of its creep life. That is, the rafted structure of NKH71 under a stress of $160 \mathrm{MPa}$ is stable for most of the creep life. On the other hand, the ratio of NKH71 under the stress of $250 \mathrm{MPa}$ increases with time. After reaching 5 at $65 \%$ of creep life, the ratio decreases. That is, the rafted structure of NKH71 under $160 \mathrm{MPa}$ is more stable than that under $250 \mathrm{MPa}$.

The dislocation density of NKH71 is plotted against the normalized creep time in Fig. 11. The density that increases rapidly up to the creep time of $3 \%$ of creep life is three times larger than the density before creep,

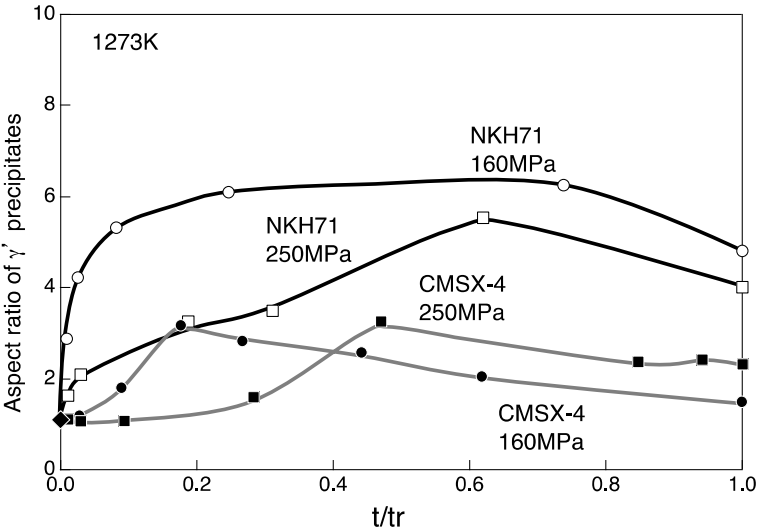

Figure 10. The change in the aspect ratios of the $\gamma^{\prime}$ precipitates of NKH71 and CMSX-4 crept at 160 and $250 \mathrm{MPa}$ with normalized creep time (ratio of time to rupture).

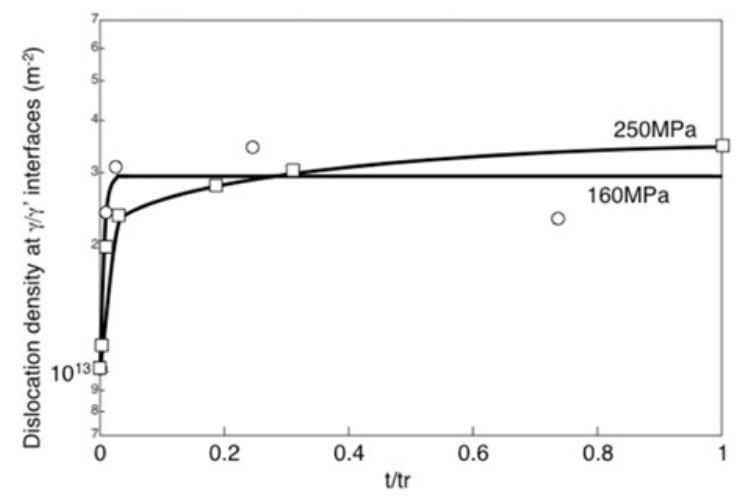

Figure 11. The change in the dislocation density at the $\gamma / \gamma^{\prime}$ interfaces of NKH71 crept at 160 and $250 \mathrm{MPa}$ with normalized creep time.

but stays unchanged until rupture. When the stress is $250 \mathrm{MPa}$, the maximum value of the density is twice larger than before creep and increases further up to rupture. These observations indicate that the dislocation density unchanging causes the stable rafted structure under $160 \mathrm{MPa}$ during creep.

It is well known that the creep resistance of $\mathrm{Ni}$ superalloys depends directly on the $\gamma$ channel width $[7,8]$. When the stress is higher than $200 \mathrm{MPa}$, the $\gamma / \gamma^{\prime}$ rafted structure is formed earlier in NKH71 than in CMSX-4. This is due to a large number of dislocations existing before creep, the number larger than that in CMSX-4. This large number of dislocations makes the width of $\gamma$ channels larger and, thus, the dislocations move more readily in NKH71. This accounts for the observation that NKH71 is weaker than CMSX-4 when the stress is large. However, when the stress is smaller, the rafted structure of NKH71 is more stable during creep. As a result, NKH71 is stronger than CMSX-4 when the stress is small.

\section{Conclusion}

The creep of a newly developed Re-free Ni-based superalloy, NKH71, is studied at $1273 \mathrm{~K}$ under the stress of 100 to $400 \mathrm{MPa}$ with SEM and TEM observations, 
in comparison with that of CMSX-4. The following conclusions are reached.

1. When the applied stress is smaller than $160 \mathrm{MPa}$, the creep life of NKH71 is longer than that of CMSX-4.

2. This result is correlated with a built-in dislocation density before creep. A large number of dislocations exist at the $\gamma / \gamma^{\prime}$ interfaces in NKH71 than in CMSX-4.

3. Further, the rafted structure, produced by creep deformation, is more stable in NKH71 than in CMSX-4 at lower stress.

4. The new alloy, NKH71, is shown to have good creep resistance even without the element $\mathrm{Re}$.

\section{References}

[1] T. Yokokawa, H. Saeki, Y. Fukuyama, T. Yoshida and H. Harada, Proc. of 10th int'l Symp. on Superalloys 2004 (2004), 859
[2] T. Hino, Y. Yoshioka, K. Nagata, H. Kashiwaya, T. Koizumi, H. Harada and T. Yamagata, Proc. 6th Liege Conf. on Mat. For Adv. Power Eng. (1988), 1129

[3] R. Darolia, D. Flahrman and R. D. Field, Proc. of 6th int'1 Symp. on Superalloys 1988 (1988), 255

[4] B. Dubiel, M. Nazmy, J. Lapin and A. CzyrskaFilemonowicz, Proc. 9th Liege Conf. on Mat. For Adv. Power Eng. (2010), 802

[5] Y. Murata, R. Hashizume, A. Yoshinari, N. Aoki, M. Morinaga and Y. Hukui, Proc. of 9h int'1 Symp. on Superalloys 2000 (2000), 285

[6] N. Miura, Y. Kondo and T. Matsuo, Proc. of 9th int'l Conf. on Creep and Fracture of Engineering Materials and Structures (2001), 437

[7] Y. Kondo and N. Miura, Advanced Materials Research 278 (2011), 138

[8] N. Miura, Y. Kondo and T. Matsuo, Tetsu-to-Hagane, 89 (2003), 1240 